\title{
Posterior Spiracles of Fourth Instar Larvae of Four Species of Phlebotomine Sand Flies (Diptera: Psychodidae) under Scanning Electron Microscopy
}

\section{Felipe Arley Costa Pessoa/ ${ }^{+}$, Raul Guerra de Queiroz, Richard Douglas Ward*}

Coordenação de Pesquisas em Ciências da Saúde, Instituto Nacional de Pesquisas da Amazônia, Caixa Postal 478, 69011-970 Manaus, AM, Brasil *School of Life Sciences, Keele University, Staffordshire, ST5 5BG, UK

In the present study, posterior spiracles of laboratory-reared fourth instar larvae of Lutzomyia longipalpis, L. migonei, L. lenti, and L. whitmani (Diptera: Psychodidae) of the State of Ceará, Brazil, were examined under scanning electron microscopy. The number of papillae of spiracles examined varied according to the species examined, but no intraspecific differences were found. The importance of this structure to sand fly larva identification and phylogeny is commented.

Key words: phlebotomine sand fly larvae - spiracle - scanning electron microscopy - Ceará - Brazil

Phlebotomine sand fly larvae are amphipneustic, having a pair of small anterior spiracles and another slightly larger pair of posterior spiracles. Although identification to species of sand fly larvae has been attempted using chaetotaxy (Ward 1976), this method is time-consuming and a number of alternative schemes have been proposed (Leite \& Williams 1996). Recently, the larval spiracles have been considered as a possible tool for immature identification and might be a useful feature for grouping species by subgenus or species-group, or to distinguish among species within the same group (Fausto et al. 1998, 1999). In the present study, posterior spiracles of three specimens of fourth instar larvae from the following laboratory colonies were examined: Lutzomyia longipalpis (progenitors from Sobral, State of Ceará, Brasil, $3^{\circ} 45^{\prime} \mathrm{S}, 40^{\circ} 5^{\prime}$ ) and $L$. migonei, L. lenti, and $L$. whitmani (progenitors from Baturité, Ceará, $4^{\circ} 20^{\circ}$ 's, $\left.38^{\circ} 55^{\prime} \mathrm{W}\right)$. They were killed in hot water $\left(70^{\circ} \mathrm{C}\right)$, fixed in 3\% glutaraldehyde and then washed thoroughly in phosphate-buffered saline, the solution being changed every $30 \mathrm{~min}$ during $6 \mathrm{~h}$. They were then fixed in osmium tetroxide, dehydrated in a series of ethyl alcohol concentrations, submitted to

\footnotetext{
${ }^{+}$Corresponding author Fax :+55-92 643.3061. E-mail: pessoa@inpa.gov.br

Received 18 August 1999

Accepted 23 February 2000
}

critical point drying in carbon dioxide and spattered with 25 MA colloidal gold. The prepared specimens were examined in a Hitachi S 4500 scanning electron microscope (SEM).

The posterior spiracles of L. longipalpis (Fig. 1) have a crater-like appearance, with a large opening in the centre and a surrounding wall bearing the papillae, separated from one another by elongated septa. Each papilla is longitudinally traversed by a slight central ridge. This feature was also observed in the three other species. A detailed description of morphology and ultrastructure of the spiracles is given by Fausto et al. (1998, 1999). In $L$. migonei (Fig. 2) the posterior spiracles are conical, with the base width about one third wider than the apex. In L. lenti (Fig. 3) and L. whitmani (Fig. 4) the posterior spiracles are cylindrical, those from the latter being more pigmented than those from all the other species examined. Table shows average number of papillae observed in these species.

With the exception of $L$. lenti, the species examined in the present study are important leishmaniasis vectors, and this is the first time that the spiracles of a species of the subgenus Nyssomyia (L. whitmani) are shown in detail. The number of papillae varied according to the species examined, but no intraspecific differences were found. Venezuelan specimens of L. longipalpis and L. migonei, however, have been reported to have a number of papillae different from Ceará specimens, and intraspecific variation (Fausto 1998) (Table). This may be due to geographic variation or could be a further evidence for L. longipalpis as a species complex (Ward et al. 1983). 


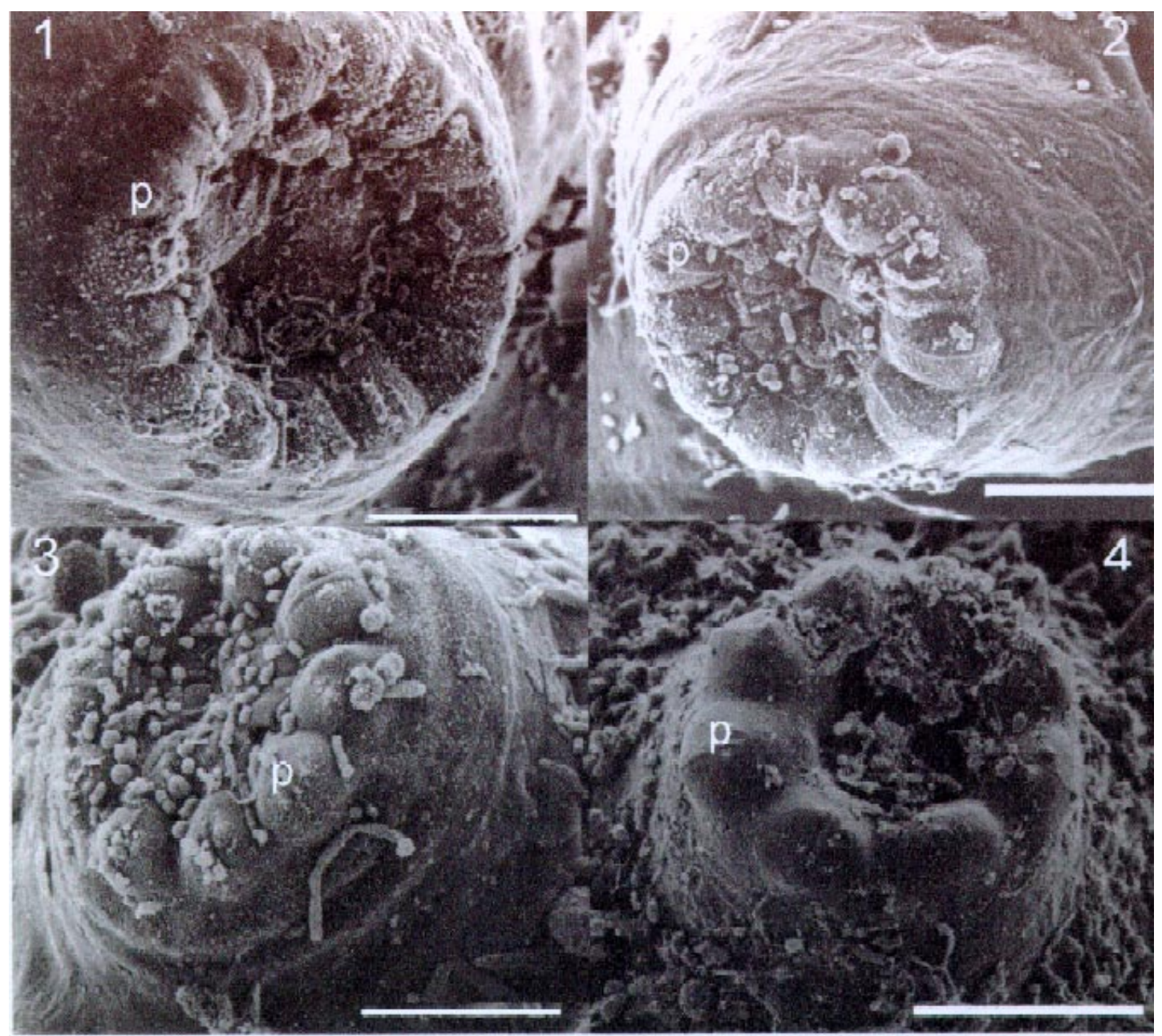

Scanning electron micrographs of posterior spiracles of fourth instar larvae. Fig. 1: Lutzomyia longipalpis $($ Bar $=9 \mu \mathrm{m})$. Fig. 2: L. migonei $(\mathrm{Bar}=10 \mu \mathrm{m})$. Fig. 3: L. lenti $(\mathrm{Bar}=7.2 \mu \mathrm{m})$. Fig. 4: L. whitmani $(\mathrm{Bar}=7.2 \mu \mathrm{m})$; p: papilla

TABLE

Average number of the papillae of the posterior larval spiracles of Lutzomyia spp.

\begin{tabular}{lcc}
\hline \multirow{2}{*}{ Species } & \multicolumn{2}{c}{ Country } \\
\cline { 2 - 3 } & Venezuela $^{a}$ & $\begin{array}{c}\text { Brazil } \\
\text { (State of Ceará) }\end{array}$ \\
\hline L. longipalpis & 18.4 & 16 \\
L. migonei & 12.4 & 10 \\
L. lenti & - & 9 \\
L. whitmani & - & 8 \\
\hline
\end{tabular}

$a$ : (Fausto et al. 1998; L. longipalpis: seven specimens; L. migonei: seven specimens); $b$ : present study (three specimens each)
Given the notorious difficulty to find Lutzomyia larvae in nature, the spiracular morphology does not appear to have much application in identification of wild specimens, but may be useful in phylogenetic studies of laboratory-reared sand flies. The papillae of the posterior spiracles can be seen under light microscopy, which could be used instead of SEM, still an expensive and unwieldy technique. This will certainly facilitate a general application of the former if these morphological structures turn out to be good taxonomic characters for sand fly larvae. However, larvae of a greater number of species will need to be examined before the taxonomic value of the papillae of the posterior spiracles can be better assessed. 


\section{REFERENCES}

Fausto AM, Feliciangeli MD, Maroli M, Mazzini M 1998. Morphological study of the larval spiracular system in eight Lutzomyia species (Diptera: Psychodidae). Mem Inst Oswaldo Cruz 93: 71-79.

Fausto AM, Taddei AR, Maroli M, Mazzini M 1999. Morphology and ultrastructure of spiracles in phlebotomine sandfly larvae. Med Vet Entomol 13: 101-109.

Leite, ACR, Williams P 1996. Description of the fourth instar larva of Lutzomyia longipalpis, under scan- ning electron microscopy. Mem Inst Oswaldo Cruz. 91: 571-578

Ward RD 1976. A revised numerical chaetotaxy for neotropical phlebotomine sandfly larvae (Diptera: Psychodidae). Systemat Ent 1:89-94.

Ward, RD, Ribeiro, AL, Ready, PD, Murtagh, A 1983. Reprodutive isolation between different forms of Lutzomyia longipalpis (Lutz \& Neiva), (Diptera: Psychodidae), the vector of Leishmania donovani chagasi Cunha \& Chagas and its significance to Kalaazar distribution in South America. Mem Inst Oswaldo Cruz 78: 269-280. 
692 Spiracles of Larvae of Sand Flies - Felipe Arley Costa Pessoa et al. 